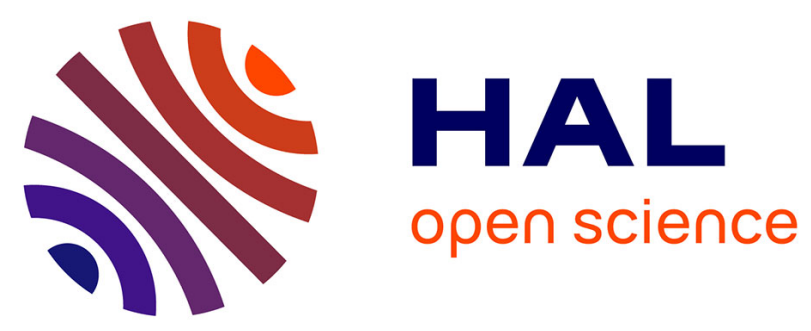

\title{
Multimodal rigid-body registration of 3D brain images using bilateral symmetry
}

\author{
Sylvain Prima, Olivier Commowick
}

\section{To cite this version:}

Sylvain Prima, Olivier Commowick. Multimodal rigid-body registration of 3D brain images using bilateral symmetry. Medical Imaging 2013: Image Processing, Feb 2013, Lake Buena Vista (Orlando Area), United States. pp.866911, 10.1117/12.2007075 . inserm-00766642

\section{HAL Id: inserm-00766642 https://www.hal.inserm.fr/inserm-00766642}

Submitted on 4 Jun 2013

HAL is a multi-disciplinary open access archive for the deposit and dissemination of scientific research documents, whether they are published or not. The documents may come from teaching and research institutions in France or abroad, or from public or private research centers.
L'archive ouverte pluridisciplinaire HAL, est destinée au dépôt et à la diffusion de documents scientifiques de niveau recherche, publiés ou non, émanant des établissements d'enseignement et de recherche français ou étrangers, des laboratoires publics ou privés. 


\title{
Multimodal rigid-body registration of 3D brain images using bilateral symmetry
}

\author{
Sylvain Prima and Olivier Commowick \\ INRIA, INSERM, VisAGeS U746 Unit/Project, F-35042 Rennes, France; \\ University of Rennes I-CNRS UMR 6074, F-35042 Rennes, France
}

\begin{abstract}
In this paper we show how to use the approximate bilateral symmetry of the brain with respect to its interhemispheric fissure for intra-subject (rigid-body) mono- and multimodal 3D image registration. We propose to define and compute an approximate symmetry plane in the two images to register and to use these two planes as constraints in the registration problem. This 6-parameter problem is thus turned into three successive 3-parameter problems. Our hope is that the lower dimension of the parameter space makes these three subproblems easier and faster to solve than the initial one. We implement two algorithms to solve these three subproblems in the exact same way, within a common intensity-based framework using mutual information as the similarity measure. We compare this symmetry-based strategy with the standard approach (i.e. direct estimation of a 6-parameter rigid-body transformation), also implemented within the same framework, using synthetic and real datasets. We show our symmetry-based method to achieve subvoxel accuracy with better robustness and larger capture range than the standard approach, while being slightly less accurate and slower. Our method also succeeds in registering clinical MR and PET images with a much better accuracy than the standard approach. Finally, we propose a third strategy to decrease the run time of the symmetry-based approach and we give some ideas, to be tested in future works, on how to improve its accuracy.
\end{abstract}

\section{INTRODUCTION}

The most popular methods for rigid-body registration of 3D images are probably those based on the maximisation of a similarity measure between the intensities of the images to align. In this context of so-called intensitybased/iconic methods, implementation choices and their impact on accuracy/precision, robustness, capture range and speed of the resulting algorithms have been extensively assessed. Most works have been dedicated to the study of:

- The design of the similarity measure. This design heavily depends on the type of registration problem. If it is monomodal (e.g. to assess longitudinal changes using MRI), then the sum of squared differences, the correlation coefficient, or any robust variant of these are proper choices. If it is multimodal (e.g. to localise activations in PET using MRI, or to compare two different MRI sequences), then the correlation ratio, ${ }^{18}$ the mutual information, ${ }^{12,25}$ or one of their numerous variants are adequate measures. A good survey on similarity measures was done by Skerl et al. ${ }^{20}$

- The computation of the similarity measure. In some cases, this can be done using the intensities directly (e.g. for the sum of squared differences) but it most often implies the preliminary computation of some probability density functions (e.g. for the mutual information). Parzen window estimates have been initially used for this purpose ${ }^{25}$ but since then most methods have used joint histograms instead, and studies have been mostly dedicated to (i) the interpolation method to build these (trilinear, partial volume, ${ }^{12}$ generalised partial volume, ${ }^{5,10,15}$ etc.) and to (ii) the binning scheme (non-equidistant binning, ${ }^{9}$ apodization and fuzzy binning, ${ }^{8}$ etc.). A good review of these issues was done by Tsao. ${ }^{23}$

- The optimisation scheme. Derivative ${ }^{25}$ as well as derivative-free techniques ${ }^{12}$ have been used, the latter being probably more popular due to easier implementation (e.g. Nelder-Mead's or Powell's algorithms). Optimisation has often been led within coarse-to-fine schemes using typically multiple resolutions/scales, down/subsampling and filtering/smoothing. A good review of these issues was done by Maes et al. ${ }^{13}$

Contact: [sprima,ocommowi] at irisa.fr

Medical Imaging 2013: Image Processing, edited by Sebastien Ourselin, David R. Haynor, Proc. of SPIE Vol. 8669, 866911 · ( 2013 SPIE · CCC code: 1605-7422/13/\$18 doi: $10.1117 / 12.2007075$

Proc. of SPIE Vol. $8669866911-1$ 
However, most intensity-based image registration methods are blind to the image content. In particular, in case of brain images, the approximate bilateral symmetry with respect to the interhemispheric fissure is typically ignored, while preliminary computation of an approximate symmetry plane in both images could potentially turn the original, 6-parameter (three rotations, three translations) registration problem into a simpler 3-parameter (one in-plane rotation and two in-plane translations) problem. We know of only one work following exactly this track, ${ }^{6}$ where a mid-sagittal plane is first estimated in both images and used as a constraint for rigid-body registration; however, in this work the computation of the two symmetry planes and that of the contrained rigidbody transformation are performed in two different manners, making it difficult to assess the real added value of this strategy. Some other works use the two symmetry planes, but only to initialise the rigid-body registration, and not to constrain it, which does not exploit the full potential of the idea, in our opinion. ${ }^{21}$ Earlier attempts in the same vein include what Maintz \& Viergever called "reductive methods", ${ }^{14}$ relying on the preliminary alignment of the centroids and principal axes of each image, as an initialisation of the registration algorithm. ${ }^{11}$ However, the computation of the centroid (based on the first-order moments of the image) and the principal axes (based on the second-order moments of the image) is highly vulnerable to deviations from perfect symmetry, which is inadequate in the context of real brain images.

In this paper, we propose to split the standard 6-parameter registration problem into three simpler 3parameter problems. Two of these consist in computing the mid-sagittal plane (3 parameters) of the brain in both images, while the third consists in computing the 3-parameter rigid transformation defined by one inplane rotation (1 parameter) and two in-plane translations (2 parameters), after the two mid-sagittal planes have been set to coincide. Contrary to what is done in the work of Favretto et al. ${ }^{6}$ these three problems are solved exactly the same way: by maximising a similarity measure between the image and its reflection with respect to the putative mid-sagittal plane (Problems 1-2) and between the two images, using the two mid-sagittal planes as constraints (Problem 3 ) and not only to initialise the registration as done by Teverovskiy \& Liu. ${ }^{21}$ Implementation choices are identical in Problems 1-2 and 3 (similarity measure, interpolation scheme to compute joint histograms, and optimisation algorithm to maximise the similarity measure). Our hope with this strategy is that each problem is easier and faster to solve than the global problem, owing to the lower dimensionality of the parameter space: the optimisation algorithm should be much less likely to be trapped in a local maximum.

We detail this two-step strategy in Section 2 (computation of the two mid-sagittal planes in Section 2.1 and constrained registration in Section 2.2). We evaluate this method and compare it to the standard one-step approach on both synthetic and real data, on which we assess the accuracy, the capture range, the robustness and the run time of the two algorithms in Section 3. We conclude and give some perspectives in Section 4.

\section{METHODS}

In this section we describe the two-step process allowing for rigid-body registration of two images $I$ and $J$. In the following, for the sake of clarity, we suppose the image and world coordinate systems to coincide (strictly speaking, it is only the case when the images are isotropic with the numbering of voxels starting from one of the image corners). For the same reason, the letter $v$ refers to both the voxel and its coordinates. The value of image $I$ at voxel $v$ is then $I(v)$, and $I$ is also termed $v \mapsto I(v)$ when needed for improved clarity; the image obtained when moving $I$ using the transformation $T$ is thus $v \mapsto I\left(T^{-1}(v)\right)$. The same notations hold for $J$ and the other images involved.

\subsection{Computation of the mid-sagittal planes in $I$ and $J$}

Functional asymmetries of the human brain have been known since the mid-nineteenth century when Paul Broca observed loss of speech in patients with a lesion in the left frontal lobe. ${ }^{2}$ Since then, these functional asymmetries have been putatively linked with morphological left-right differences, starting with the landmark paper of Geschwind \& Levitsky showing such asymmetries in the temporal lobes. ${ }^{7}$ Still, the human brain exhibits a striking rough bilateral symmetry with respect to a so-called mid-sagittal plane.

Many methods have been proposed to properly define and compute this plane in a brain image, and then to resample the image to make the plane coincide with the centre of the image volume; this way, it is ensured that homologous anatomical structures in the two hemispheres are correctly displayed in the same axial or coronal slice. There are mostly two classes of methods for this purpose. In the first class, the plane is defined as the

Proc. of SPIE Vol. $8669866911-2$ 
one best fitting the interhemispheric fissure, ${ }^{3}$ or as the one giving a cross-section with optimal properties, e.g. minimal entropy of the image intensities ${ }^{24}$ or minimal area of the corpus callosum. ${ }^{4}$ In the second class, the plane is defined as that cutting the brain in two parts as similar as possible; ${ }^{1}$ in other words, one looks for a plane (or equivalently, a reflection symmetry) which maximises the similarity between the brain and itself when mirrored about this plane.

This second viewpoint is especially attractive as it amounts to computing an optimal transformation (a reflection symmetry) linking two images, and thus one can resort to the many methods previously defined for rigid-body or affine registration of images, and in particular the most popular intensity-based methods. This is the track we follow here to define and compute the mid-sagittal planes $P$ (resp. $Q$ ) of the brain in $I$ (resp. $J$ ).

We simply define the plane $P$ as that maximising the similarity between:

$$
v \mapsto I(v) \text { and } v \mapsto I\left(S_{P}(v)\right),
$$

where $S_{P}$ is the reflection across $P$. This plane $P$ is parametrized by a unit vector $\vec{n}$ (represented by two angles $\alpha$ and $\beta$ in a spherical coordinate system) and its distance $d$ to the origin of the coordinate system; then the reflection $S_{P}$ is simply:

$$
v \mapsto S_{P}(v)=\left(I_{3}-2 \vec{n} \vec{n}^{T}\right) v+2 d \vec{n},
$$

where $I_{3}$ is the $3 \times 3$ identity matrix. The same optimisation is performed to compute $Q$. Further implementation details (similarity measure, interpolation technique, optimisation algorithm) are provided in Section 2.3.

\subsection{Constrained rigid-body registration of $I$ and $J$}

One can easily see that, when applied to $J$, the rigid-body transformation $\left(S_{P} \circ S_{Q}\right)^{1 / 2}$ aligns $Q$ with $P .{ }^{17}$ Note that this transformation is either a pure rotation (if $P$ and $Q$ intersect) or a pure translation (if $P$ and $Q$ are parallel). If one assumes that the two planes cut the brain at the exact same anatomical location in images $I$ and $J$, then one can also suppose that the images:

$$
v \mapsto I(v) \text { and } v \mapsto J\left(\left(S_{P} \circ S_{Q}\right)^{-1 / 2}(v)\right)
$$

are aligned, up to a constrained rigid-body transformation, whose axis is perpendicular to $P$ and whose translation is parallel to $P$. In other words, this constrained transformation $R_{P}$ can be simply parametrized by one angle $\gamma$ for the rotation and two real numbers $t$ and $u$ for the translation. We define and compute this constrained transformation $R_{P}$ ( $P$ and $Q$ being fixed) as the one best superposing:

$$
v \mapsto I(v) \text { and } v \mapsto J\left(\left(S_{P} \circ S_{Q}\right)^{-1 / 2} \circ R_{P}^{-1}(v)\right)
$$

Further implementation details (similarity measure, interpolation technique, optimisation algorithm) are provided in Section 2.3.

\subsection{Implementation details}

The three above-mentioned problems (Sections 2.1 and 2.2) are solved exactly the same way, using:

- mutual information as the similarity measure ${ }^{12}$

- partial volume interpolation to compute the joint histogram ${ }^{12}$

- NEWUOA to maximise the similarity measure computed from the joint histogram ${ }^{16}$

- $l$-level multiresolution scheme ${ }^{13}$ 
For these three subproblems, we compute the optimal transformation over $l=3$ pyramid levels. At each pyramid level, we used the following NEWUOA search radius: $\alpha=5$ degrees, $\beta=5$ degrees, $d=2$ voxels (Sec. 2.1), $\gamma=5$ degrees, $t=2$ voxels, and $u=2$ voxels (Sec. 2.2).

Computing the mid-sagittal plane in $I$ and $J$ is typically a monomodal problem, while registering $I$ and $J$ is not (or, at least, not necessarily). Thus one could have chosen a simpler similarity measure for the first task, such as the sum of squared differences. However, owing to the fact that our goal is to assess this two-step method in comparison with the standard one-step approach, it is desirable to use the same similarity measure everywhere. Mutual information is the most common choice, and in addition it is much more robust than the sum of squared differences, even in monomodal problems. Robustness is especially important here, as the brain is only approximately symmetrical, and thus there are necessarily outliers (lesions, tumours, "normal" inter-hemispheric differences, etc.) to be dealt with in Problems 1-2.

\section{VALIDATION AND RESULTS}

We evaluate our registration method which uses bilateral symmetry (termed REG-SYM) and compare it with the standard strategy consisting in the direct computation of the 6-parameter rigid-body transformation (termed REG-DIR), implemented in the same framework as REG-SYM (cf. Sec. 2.3). We also assess a third strategy, also implemented in the same framework, in which we use only the $2 \mathrm{D}$ sections cut by the planes $P$ and $Q$ in $I$ and $J$ rather than their whole 3D volumes. These sections are related to each other through the same constrained transformation as the 3D volumes, thus one can expect this REG-CUT algorithm to show a decreased run time compared to REG-SYM.

For these three registration strategies, we evaluate the accuracy, robustness, capture range and run time, on three datasets composed of two images $I$ and $J$ each. Random rigid-body displacements are simulated for Datasets 1-2, while the ground truth transformation is known for Dataset 3.

\subsection{Validation setting}

Random experiments. For Datasets 1-2, in which $I$ and $J$ are initially aligned, we generate 150 random rigidbody transformations $T$ (with translations in the range $[0,15] \mathrm{mm}$ and rotations in the range $[0,50]$ degrees around each of the three image axes), and then we apply $T^{1 / 2}$ to the first image $I$, and $T^{-1 / 2}$ to the second image $J$. This gives two misaligned images defined as, respectively, $v \mapsto \tilde{I}(v)=I\left(T^{-1 / 2}(v)\right)$ and $v \mapsto \tilde{J}(v)=J\left(T^{1 / 2}(v)\right)$. The resampling is done using trilinear interpolation. We also add white Gaussian noise (standard deviation: $10 \%$ of the average image signal) to both $\tilde{I}$ and $\tilde{J}$.

Warping indices. Given there are $n$ voxels in each of the two image grids, we define the initial warping index as $\omega_{i}=\sum_{v}\|T(v)-v\| / n ; \omega_{i}$ measures the misalignment of $\tilde{I}$ (considered as the reference image) and $\tilde{J}$ (considered as the floating image). After registration of $\tilde{J}$ onto $\tilde{I}$, giving the transformation $F$, we define the final warping index as $\omega_{f}=\sum_{v}\|T(v)-F(v)\| / n ; \omega_{f}$ measures the registration error. ${ }^{22}$

Evaluation metrics. The registration is considered a success if $\omega_{f}$ is lower than the voxel size. We define the capture range of the algorithm as the maximum $\omega_{i}$ for which it succeeds. We define its accuracy as the average $\omega_{f}$ when it succeeds. We define its robustness as the percentage of random experiments being a success. We also report its average run time (on one core of a Xeon $3.0 \mathrm{GHz} \mathrm{PC}$ ).

Dataset 1. The first dataset comes from the BrainWeb MRI simulator*. We consider the T1-weighted vs T2-weighted MRI (size $181 \times 217 \times 181$, voxel size $1 \mathrm{~mm}^{3}$ ) with no noise (as it is added in our experiments).

Dataset 2. The second dataset is a real dataset acquired on a healthy subject. It is a dual echo T2/PD sequence ( size $192 \times 256 \times 44$, voxel size $1 \times 1 \times 3 \mathrm{~mm}^{3}$ ). Due to interleaved acquisition, the two images are natively aligned.

\footnotetext{
*http://www.bic.mni.mcgill.ca/brainweb
} 
Dataset 3. The last dataset is a real clinical dataset coming from the R.I.R.E initiative for the comparison of multimodal registration algorithms ${ }^{\dagger}$. It consists of an MR image (size $256 \times 256 \times 26$, voxel size $1.25 \times 1.25 \times 4 \mathrm{~mm}^{3}$ ) and of a PET image (size $128 \times 128 \times 16$, voxel size $2.59 \times 2.59 \times 8 \mathrm{~mm}^{3}$ ). These two images are acquired with fiducial markers providing objective landmark points (available from the website) from which the true transformation is computed.

\subsection{Results}

The results on Datasets 1-2 are reported in Tables 1-2, while Fig. 1 shows an example of registration of a T2weighted onto a PD-weighted MR image (Dataset 1) and Fig. 2 shows the results of the registration of the PET image onto the MR image (Dataset 3).

On Datasets 1-2 (cf. Tab. 1-2 and Fig. 1), REG-DIR is on average 1.35 times faster than REG-SYM, which was to be expected, as 9 parameters are estimated in the latter and only 6 in the former. However, this increase of run time is largely compensated by the fact that the output of REG-SYM is more than just the two images aligned with each other: the two images can also be displayed in a proper coordinate system, with the mid-sagittal plane located at the centre of the image volumes, as shown in Fig. 1(c-f). In addition, when several images have to be registered on the same template image (e.g. when comparing follow-up scans to a baseline scan), the mid-sagittal plane of the latter is computed only once, which results in an overall decreased run time for the whole process. REG-DIR is also on average between 2 (on Dataset 2) and 4 (on Dataset 1) times more accurate than REG-SYM, which was also to be expected, as splitting the global problem into three subproblems was likely to lead to some error propagation. However, REG-SYM is on average about $10 \%$ more robust than REG-DIR, which means that it achieves subvoxel accuracy more often than REG-DIR, compared to which it also shows a slightly larger capture range; this confirms that in some cases the one-step registration strategy fails to find the correct transformation, probably due to the higher dimensional parameter space, while the twostep registration strategy succeeds. In terms of run time, the third algorithm REG-CUT is a nice alternative to REG-SYM, as it shares on average the same capture range, accuracy and robustness, while being as fast as REG-DIR.

On Dataset 3 (cf. Fig. 2), for which the initial warping index $\omega_{i}$ is $27.9 \mathrm{~mm}$, REG-SYM achieves very high accuracy $\left(\omega_{f}=3.8 \mathrm{~mm}\right)$ compared to the large voxel size of the images (and especially that of the PET image: $\left.2.59 \times 2.59 \times 8 \mathrm{~mm}^{3}\right)$, closely followed by REG-CUT $\left(\omega_{f}=5.6 \mathrm{~mm}\right)$, while REG-DIR is much less accurate $\left(\omega_{f}=22.0 \mathrm{~mm}\right)$.

\begin{tabular}{|c|c|c|c|c|}
\hline & Cap. range & Acc. & Rob. & Time \\
\hline REG-DIR & 137.4 & $0.07 \pm 0.10$ & 88.0 & 323.5 \\
\hline REG-SYM & 142.9 & $0.27 \pm 0.08$ & 97.3 & 457.2 \\
\hline REG-CUT & 137.4 & $0.28 \pm 0.08$ & 94.7 & 330.8 \\
\hline
\end{tabular}

Table 1. Experiments on the BrainWeb simulated dataset. Reports of capture range (mm), accuracy (mm), robustness (\%) and average computation time (seconds) for the 150 simulated transformations on Dataset 1.

\begin{tabular}{|c|c|c|c|c|}
\hline & Cap. range & Acc. & Rob. & Time \\
\hline REG-DIR & 164.2 & $0.33 \pm 0.16$ & 80.7 & 172.9 \\
\hline REG-SYM & 164.2 & $0.69 \pm 0.26$ & 92.0 & 222.5 \\
\hline REG-CUT & 164.2 & $0.71 \pm 0.28$ & 91.3 & 163.5 \\
\hline
\end{tabular}

Table 2. Experiments on the T2/PD real dataset. Reports of capture range (mm), accuracy (mm), robustness (\%) and average computation time (seconds) for the 150 simulated transformations on Dataset 2.

\footnotetext{
${ }^{\dagger}$ http://www.insight-journal.org/rire
} 


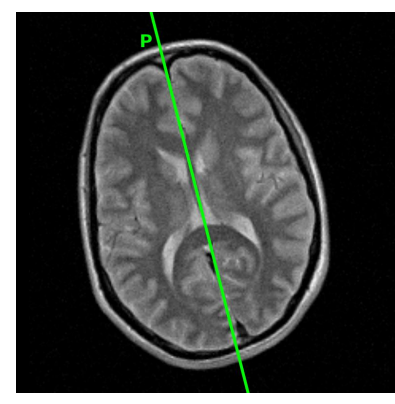

(a)

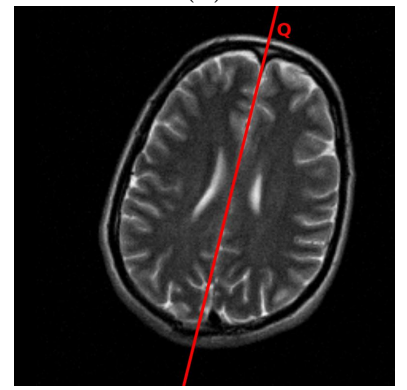

(d)

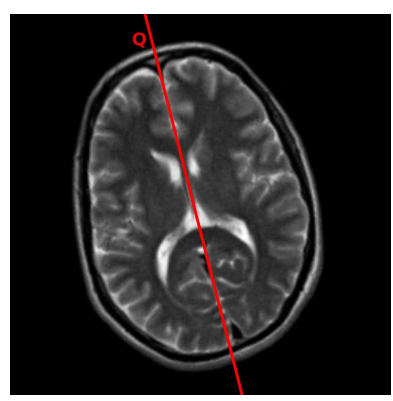

(b)

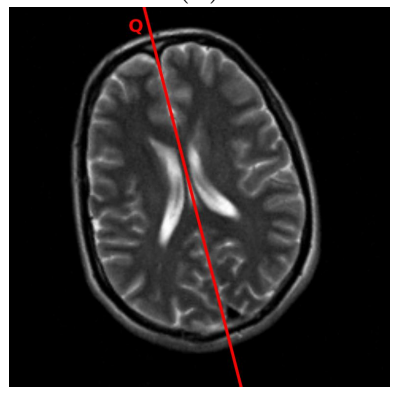

(e)

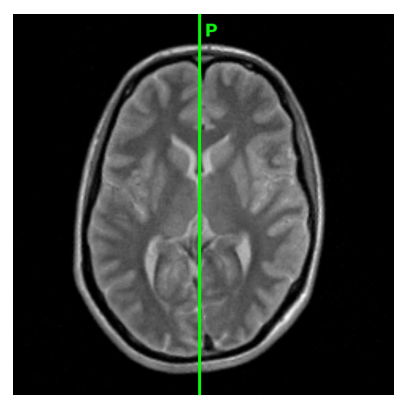

(c)

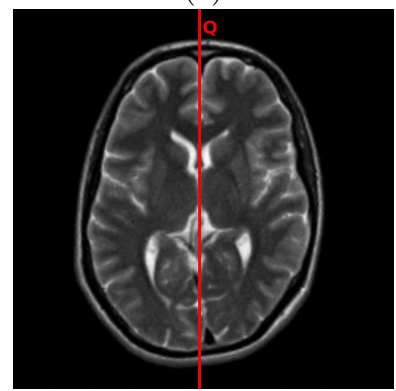

(f)

Figure 1. Registration results on real T2/PD images with simulated mismatch. Illustration of the symmetrybased registration strategy: (a) PD-weighted MR reference image ( $P$ is the estimated mid-sagittal plane); (d) T2-weighted MR floating image ( $Q$ is the estimated mid-sagittal plane); (e) floating image transformed so that $Q$ is aligned with $P$; (b) floating image aligned on reference image using the hypothesis that $P=Q$; (c) and (f) show both images aligned with each other, and displayed so that the mid-sagittal plane $P=Q$ is located at the centre of the image volumes. This allows one to visually assess the Yakovlevian torque of the brain: the left occipital lobe appears wider and protrudes more posteriorly compared to the right; this asymmetry, of a lesser extent, is reversed in the frontal lobes.

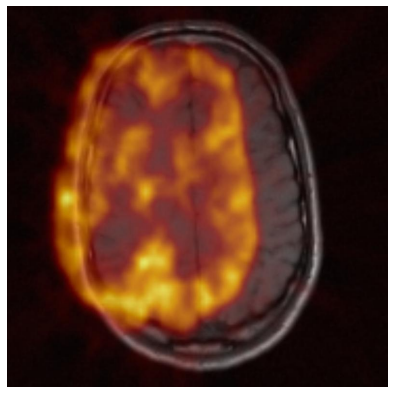

(a)

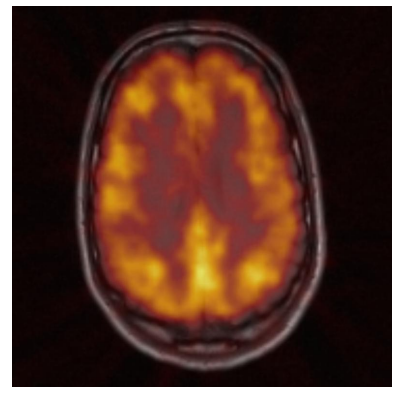

(b)

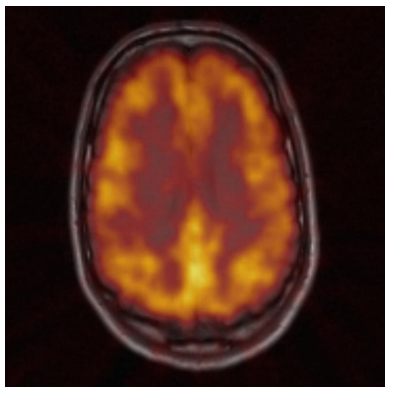

(c)

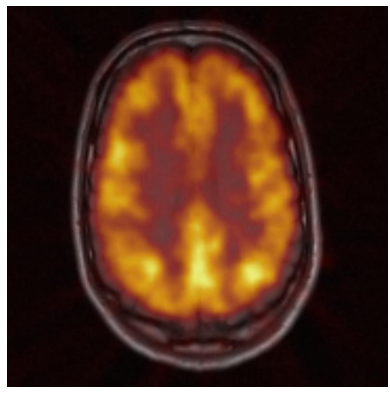

(d)

Figure 2. Registration results on real MR/PET images. This figure presents the initial configuration of MR and PET images before registration (a). Then, from left to right: PET image registered on the MR image using (b) REG-DIR, (c) REG-SYM, and (d) REG-CUT. 


\section{CONCLUSION AND PERSPECTIVES}

In this paper we presented a method to use the approximate bilateral symmetry of the human brain to perform intra-subject, multimodal, rigid-body registration. The mid-sagittal plane is estimated in both images to register, and is used as a constraint to solve the registration problem. Estimation of the plane and computation of the constrained rigid-body transformation are performed the same way, by maximising the same intensity-based similarity measure.

We showed this strategy to be more robust, with a larger capture range, than the standard strategy where an optimal global rigid-body transformation is computed directly without using symmetry. This suggests that in some cases the latter strategy fails to find the correct transformation, probably due to the higher dimensional parameter space, while the former succeeds.

The symmetry-based strategy appears to be slightly slower and less accurate than the standard one; this was to be expected, as 9 parameters are estimated in the former instead of 6 in the latter, and as there is some error propagation in the former. However, the increased run time can be largely compensated in some problems where one wants to register several images to a template/baseline image; using a computationally less expensive monomodal similarity measure to compute the two mid-sagittal planes could be another option to investigate for the same purpose. Moreover, the added value of the symmetry-based algorithm is that it also provides a consistent display of the images, allowing for morphological asymmetries to be visually assessed in a straightforward manner.

In addition to this symmetry-based method, we proposed a third strategy in which the constrained registration is performed using only one $2 \mathrm{D}$ slice, which results in a run time comparable with that of the standard strategy, with the same robustness and capture range as the symmetry-based strategy. Here, we chose the 2D slice as being that cut by the mid-sagittal plane; one could also use the average of the slices parallel to this plane. ${ }^{19}$ As for the reduced accuracy, one could imagine a fourth strategy in which the mid-sagittal plane would be computed at low resolution levels, and used to initialise the standard algorithm at high resolution levels; that could result in an algorithm with high accuracy, large capture range, high robustness, and low run time. Another line of research would be to investigate the use of symmetry in inter-subject registration problems, for instance in atlas construction.

\section{REFERENCES}

[1] B.A. Ardekani, J. Kershaw, M. Braun, and I. Kanno. Automatic detection of the mid-sagittal plane in 3-D brain images. IEEE Transactions on Medical Imaging, 16(6):947-952, December 1997.

[2] P. Broca. Sur le siège de la faculté du langage articulé. Bulletins de la Société d'Anthropologie, 6:377-393, 1865.

[3] M.E. Brummer. Hough transform detection of the longitudinal fissure in tomographic head images. IEEE Transactions on Medical Imaging, 10(1):74-81, March 1991.

[4] N. Changizi, G. Hamarneh, O. Ishaq, A. D. Ward, and R. Tam. Extraction of the plane of minimal crosssectional area of the corpus callosum using template-driven segmentation. In 11th International Conference on Medical Image Computing and Computer-Assisted Intervention, MICCAI'2010, volume 6363 of LNCS, pages 17-24, September 2010.

[5] S. Darkner and J. Sporring. Generalized partial volume: an inferior density estimator to Parzen windows for normalized mutual information. In 22nd International Conference on Information Processing in Medical Imaging, IPMI'11, volume 6801 of LNCS, pages 436-447, July 2011.

[6] F.O. Favretto, F. Bergo, and A.X. Falcao. A fast and automatic method for 3D rigid registration of MR images of the human brain. In 21st Brazilian Symposium on Computer Graphics and Image Processing, SIBGRAPI'08, pages 121-128, October 2008.

[7] N. Geschwind and W. Levitsky. Human brain: left-right asymmetries in temporal speech region. Science, 161(837):186-167, July 1968.

[8] M. Jenkinson, P. Bannister, M. Brady, and S. Smith. Improved optimization for the robust and accurate linear registration and motion correction of brain images. NeuroImage, 17(2):825-841, October 2002. 
[9] Z.F. Knops, J.B.A. Maintz, M.A. Viergever, and J.P.W. Pluim. Normalized mutual information based registration using k-means clustering and shading correction. Medical Image Analysis, 10(3):432-439, 2006.

[10] D. Loeckx, F. Maes, D. Vandermeulen, and P. Suetens. Comparison between Parzen window interpolation and generalised partial volume estimation for nonrigid image registration using mutual information. In 3rd International Workshop on Biomedical Image Registration, WBIR'2006, volume 4057 of LNCS, pages 206-213, July 2006.

[11] Zhentai Lu, Minghui Zhang, Qianjin Feng, Pengcheng Shi, and Wufan Chen. Medical image registration based on equivalent meridian plane. In 4th International Conference on Image Analysis and Recognition, ICIAR'200\%, volume 4633 of LNCS, pages 982-992, August 2007.

[12] F. Maes, A. Collignon, D. Vandermeulen, G. Marchal, and P. Suetens. Multimodality image registration by maximization of mutual information. IEEE Transactions on Medical Imaging, 16(2):187-198, April 1997.

[13] F. Maes, D. Vandermeulen, and P. Suetens. Comparative evaluation of multiresolution optimization strategies for multimodality image registration by maximization of mutual information. Medical Image Analysis, 3(4):373-386, December 1999.

[14] J.B.A. Maintz and M.A. Viergever. A survey of medical image registration. Medical Image Analysis, 2(1):1-36, March 1998.

[15] Hua mei Chen and P.K. Varshney. Mutual information-based CT-MR brain image registration using generalized partial volume joint histogram estimation. IEEE Transactions on Medical Imaging, 22(9):1111-1119, September 2003.

[16] M.J.D. Powell. The NEWUOA software for unconstrained optimization without derivatives. In G. Pillo, M. Roma, and Panos Pardalos, editors, Large-Scale Nonlinear Optimization, volume 83 of Nonconvex Optimization and Its Applications, pages 255-297. Springer, 2006.

[17] S. Prima, S. Ourselin, and N. Ayache. Computation of the mid-sagittal plane in 3-D brain images. IEEE Transactions on Medical Imaging, 21(2):122-138, February 2002.

[18] A. Roche, G. Malandain, and N. Ayache. Unifying maximum likelihood approaches in medical image registration. International Journal of Imaging Systems and Technology, 11(1):71-80, 2000.

[19] R. Shams, R.A. Kennedy, P. Sadeghi, and R. Hartley. Gradient intensity-based registration of multi-modal images of the brain. In 11th International Conference on Computer Vision, ICCV'07, pages 1-8, October 2007.

[20] D. Skerl, B. Likar, and F. Pernus. A protocol for evaluation of similarity measures for rigid registration. IEEE Transactions on Medical Imaging, 25(6):779-791, June 2006.

[21] L. Teverovskiy and Y. Liu. Truly 3D midsagittal plane extraction for robust neuroimage registration. In 3rd IEEE International Symposium on Biomedical Imaging: From Nano to Macro, ISBI'06, April 2006.

[22] P. Thévenaz, U.E. Ruttimann, and M. Unser. A pyramid approach to subpixel registration based on intensity. IEEE Transactions on Image Processing, 7(1):27-41, January 1998.

[23] J. Tsao. Interpolation artifacts in multimodality image registration based on maximization of mutual information. IEEE Transactions on Medical Imaging, 22(7):854-864, July 2003.

[24] I. Volkau, K.N. Bhanu Prakash, A. Ananthasubramaniam, A. Aziz, and W. L. Nowinski. Extraction of the midsagittal plane from morphological neuroimages using the Kullback-Leibler's measure. Medical Image Analysis, 10(6):863-874, December 2006.

[25] W.M. Wells, P. Viola, H. Atsumid, S. Nakajimae, and R. Kikinis. Multi-modal volume registration by maximization of mutual information. Medical Image Analysis, 1(1):35-51, March 1996. 